\title{
Regularized representation of bacterial hydrodynamics
}

\author{
Kenta Ishimoto, ${ }^{1, *}$ Eamonn A. Gaffney, ${ }^{2, \dagger}$ and Benjamin J. Walker ${ }^{2,+}$ \\ ${ }^{1}$ Research Institute for Mathematical Sciences, Kyoto University, Kyoto 606-8502, Japan \\ ${ }^{2}$ Wolfson Centre for Mathematical Biology, Mathematical Institute, University of Oxford, \\ Oxford OX2 6GG, United Kingdom
}

(Received 25 March 2020; accepted 30 July 2020; published 8 September 2020)

Fluid flows induced by a flagellated bacterial swimmer are often modeled as a simple force dipole, valid in the far field. Such representations neglect the inherent rotation of these bacteria as they swim, driven by a spinning helical flagellum or fascicle. Here, we present a refined swimmer representation that makes use of regularized singularities, retaining simplicity while capturing details of the complex flow field near the swimmer that have previously been absent from basic models. We illustrate the significance of this representation via a study of bacterial predator-prey dynamics, highlighting the importance of detailed hydrodynamics in models of bacterial interactions and bacterial active matter.

DOI: 10.1103/PhysRevFluids.5.093101

\section{INTRODUCTION}

Distilling simplicity from complexity is an extensive goal of scientific study. However, in the microscale world of active matter and cellular swimming, simplicity is regularly ad hoc and not uniformly valid, with the basic and intuitive reductions often neglecting significant aspects of the dynamics. One such example is the representation of force-free swimmers purely as force dipoles, popularized by computational necessity in the 20th Century and summarized in the extensive review of Lauga and Powers [1]. Though the foundation of innumerable insightful studies into swimming at the microscale, such a representation potentially neglects extensive detail in the near-field of the microswimmer. Reliably capturing only the far-field flows, these models remain in widespread use, even though hydrodynamic interactions between swimmers at closer scales can exhibit sensitivity to both individual geometry and modality of locomotion [2-4], in addition to being of great interest in active matter physics [5-7].

As a particular illustration, flagellated bacterial microswimmers, such as Escherichia coli, Pseudomonas aeruginosa, Rhodobacter spheroides, and Bacillus subtilis, utilize the rotation of one or more long slender flagella to achieve motility, with the multiple flagella of E. coli and $B$. subtilis bundled into a single helical fascicle that resembles the lone helical flagellum of $R$. spheroides or $P$. aeruginosa. While large-scale collective motion has been observed to generate turbulent flows at high concentrations in the case of B. subtilis [8], at a more basic level the rotary motion generally induces complex spiral-like velocity fields around the swimmers. However, this

\footnotetext{
*ishimoto@kurims.kyoto-u.ac.jp

†gaffney@maths.ox.ac.uk

Corresponding author: benjamin.walker@maths.ox.ac.uk
}

Published by the American Physical Society under the terms of the Creative Commons Attribution 4.0 International license. Further distribution of this work must maintain attribution to the author(s) and the published article's title, journal citation, and DOI. 
defining characteristic of bacterial flows is neglected in their entirety by minimalistic force-dipole representations. Despite this significant absence of detail, and potential qualitative impacts on resulting swimming behaviors [9], these force-dipole models are frequently used in studies of bacterial swimming [10-18].

There are a multitude of more refined models of helically driven swimmers, from popular regularized Stokeslet methods to fully resolved boundary integral formulations [19-23], each balancing accuracy with tractability. Most reminiscent of simple force dipole models, the rotational motion of torque-free swimmers may be incorporated into singularity representations via the inclusion of torque dipoles, also known as rotlet dipoles, which can appear as higher-order singularities in the far-field multipole expansion of the flow generated by a swimmer. Such terms are noted by Lopez and Lauga [9] to be the source of the circular motion of cells near boundaries, and have been used to study droplets of active fluid to reveal rich and varied behaviors [24]. However, the efficacy of representing near-field flows via rotlet dipoles is yet to be evaluated in significant detail. Hence, the primary aim of this study will be to construct a representation of bacterial hydrodynamics that incorporates the characteristics of rotlet dipole flows, making use of the regularized singularities of Cortez [25] to afford additional computational flexibility and accuracy.

Despite their noted importance in cell-surface interactions, the impact of the rotational flows induced by rotlet dipoles on cell-cell interactions remains largely untested. Furthermore, in the context of bacterial swimming, the importance of hydrodynamic cell-cell interactions is not entirely resolved. For example, the detailed study of Drescher et al. [26] suggests that the effects of rotational noise dominate swimmer interactions in the near-field, though notably makes use of a force dipole model of bacterial swimming, with rotational effects absent. Somewhat contrastingly, it is suggested that the hydrodynamic no-slip condition contributes significantly to the successful surface entrainment of particles in the vicinity of swimmers [27-30]. Therefore, with both rotational diffusion and hydrodynamics potentially relevant to swimmer-swimmer interactions, a further objective of this study is to perform minimalistic illustrative simulations of a model in silico system of interacting bacteria to determine the extent to which these distinct components impact on cell movement and potential swimmer-swimmer collisions. We do this with particular reference to the biological context of contact-mediated bacterial predation, a phenomenon in which one species predates upon another via surface contact, for example in the case of the predation of Escherichia coli by the aptly named Bdellovibrio bacteriovorus [31,32]. We direct the interested reader to the recent reviews of Pérez et al. [33] and Negus et al. [34] for a comprehensive summary of bacterial predation.

Hence, in this work we will firstly aim to present a simple representation of helically driven swimmers, focusing on archetypal bacterial morphology and utilizing the regularized singularities of Cortez [25] to extensively capture the details of bacterial swimming. In particular, we will incorporate the rapid rotational motion of the helix via discrete regularized rotlets, and evaluate the efficacy of near-field flow approximation using these regularized singularities in comparison to both singular and regularized force dipole models. We will then seek to exemplify the significance of this representation to bacterial hydrodynamics by briefly considering idealized predatory bacterial dynamics, adapting the methodology of Ishimoto and Gaffney [35] to simulate the pairwise swimming of model predator and prey bacteria. We will examine the effects of including both hydrodynamic interactions and rotational diffusion, aiming to quantify the significance of each of these factors on overall swimmer-swimmer dynamics.

\section{METHODS}

\section{A. Regularized singularities of Stokes flow}

The fluid flows generated by the microswimming of various individual bacteria are governed by the well-known Stokes equations, which for Newtonian stress tensor $\boldsymbol{\sigma}$ and applied force $\boldsymbol{F}$ may be written as

$$
\nabla \cdot \sigma+\boldsymbol{F}=\mathbf{0}
$$


accompanied by force and torque-free conditions due to a negligibly small Reynolds number. Associated with these linear equations are fundamental singularities, with perhaps the most basic being the Stokeslet and the rotlet, corresponding to the action of a point force and torque on the fluid, respectively. A regularized analog of the Stokeslet [25], which enables simple computation of the associated flow fields owing to its nonsingular construction, has previously been used to represent the swimming of another model organism, the human spermatozoon [36]. In this work we will attempt to represent the flow fields around a bacterium using so-called regularized Stokeslets and, in addition, regularized rotlets, which, when placed at a location $\boldsymbol{x}_{0}$, generate flows at a field point $\boldsymbol{x}$ given by $\boldsymbol{u}(\boldsymbol{x})=\boldsymbol{f} \cdot \boldsymbol{G}_{\epsilon_{S}}\left(\boldsymbol{x}, \boldsymbol{x}_{0}\right)$ and $\boldsymbol{u}(\boldsymbol{x})=\boldsymbol{m} \times \boldsymbol{T}_{\epsilon_{R}}\left(\boldsymbol{x}, \boldsymbol{x}_{0}\right)$, where $\boldsymbol{G}_{\epsilon_{S}}$ and $\boldsymbol{T}_{\epsilon_{R}}$ are the regularized Stokeslet and rotlet and $\boldsymbol{f}$ and $\boldsymbol{m}$ are their respective force and torque strengths. Explicitly, here we take

$$
\boldsymbol{G}_{\epsilon_{S}}=\frac{\left(r^{2}+2 \epsilon_{S}^{2}\right) \boldsymbol{I}+\boldsymbol{r} \boldsymbol{r}}{\left(r^{2}+\epsilon_{S}^{2}\right)^{3 / 2}}, \quad \boldsymbol{T}_{\epsilon_{R}}=\frac{\left(2 r^{2}+5 \epsilon_{R}^{2}\right) \boldsymbol{r}}{\left(r^{2}+\epsilon_{R}^{2}\right)^{5 / 2}},
$$

where $\boldsymbol{r}=\boldsymbol{x}-\boldsymbol{x}_{0}, \boldsymbol{r}=|\boldsymbol{r}|$, and $\epsilon_{S}, \epsilon_{R}>0$ are the regularization parameters associated with the regularized Stokeslet and rotlet, respectively. These expressions for the regularized Stokeslet and rotlet correspond to taking $\boldsymbol{F}=\boldsymbol{f} \phi_{\epsilon_{S}}(\boldsymbol{r})$ and $\boldsymbol{F}=\boldsymbol{\nabla} \times\left[\boldsymbol{m} \phi_{\epsilon_{R}}(\boldsymbol{r})\right]$ in Eq. (1), respectively, where

$$
\phi_{\epsilon}(\boldsymbol{r})=\frac{15 \epsilon^{4}}{8 \pi\left(r^{2}+\epsilon^{2}\right)^{7 / 2}} .
$$

By linearity, the flow due to a collection of these regularized point forces and torques is simply the sum of their individual flows.

\section{B. Flow-field simulation}

We compute, to high accuracy, the motion of a bacterial swimmer using the boundary element method [37], as implemented and verified by Walker et al. [38], imposing force and torque-free conditions on the virtual swimmer. We adopt the approximate morphology of the monoflagellate $P$. aeruginosa [39], representing the body as a spherocylinder and the flagellum as a rigid helix rotating with constant angular velocity relative to the swimmer body, with the details of the flagellar shape as in Dasgupta et al. [39], Ishimoto [40]. In dimensionless units, the flagellum is taken to be of unit length and radius 0.003 , with the cell body of length 0.3 and radius 0.1 . Computing the flow field around the swimmer in the laboratory frame at sample points that translate with the linear motion of the bacterium, as measured at the body-flagellum junction, we average the flow velocity $\boldsymbol{u}$ over multiple periods of the cyclical motion of the helix. Denoting this time-averaged flow field by $\boldsymbol{u}_{a}$, we show a slice of the flow and accompanying sample streamlines in Figs. 1(a) and 1(d), and 1(g) from which the clear influence of the rapidly rotating helix can be seen. These flow profiles are qualitatively different to the symmetric flow fields generated by simple force-dipole representations of swimmers, with the apparent structural complexity of the cyclical streamlines evident here.

\section{Simulation of predator-prey interactions}

We will simulate the three-dimensional swimming of a pair of model swimmers by adapting the methodology of Ishimoto and Gaffney [35] to include the contributions of regularized rotlets, and we will include the effects of rotational diffusion where specified. For a full description of the swimming model and implementation we direct the reader to the publication of Ishimoto and Gaffney [35], and we briefly summarize the approach here. An individual swimmer is represented by a collection of regularized singularities and moves with a prescribed linear velocity, which for a given timescale is computed as a byproduct of the boundary element method flow-field computation. This velocity, denoted $\boldsymbol{U}^{\text {self }}$, is augmented by the flow-field contribution of the other individual, exploiting the linearity of Stokes equations. Explicitly, the centroid of the $k$ th swimmer evolves 


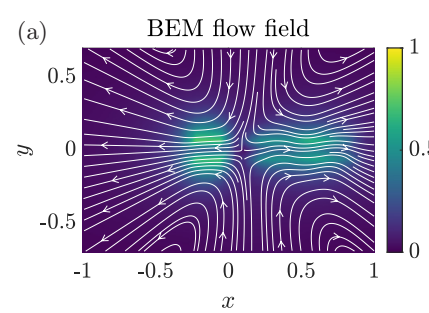

(d)

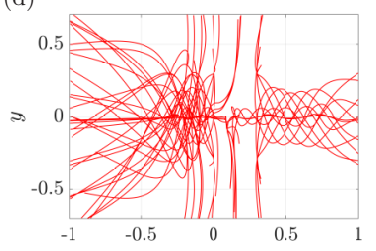

(g)

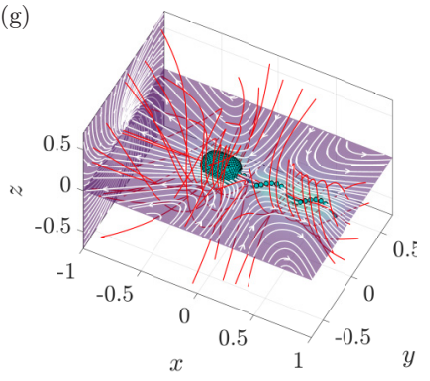

(b)

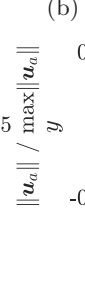

Fitted representation

(e)
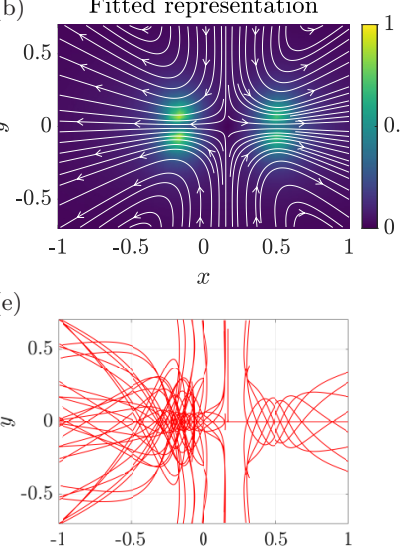

(h)

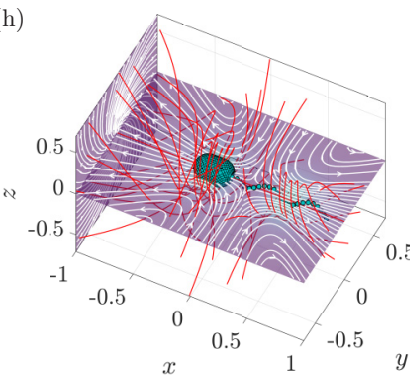

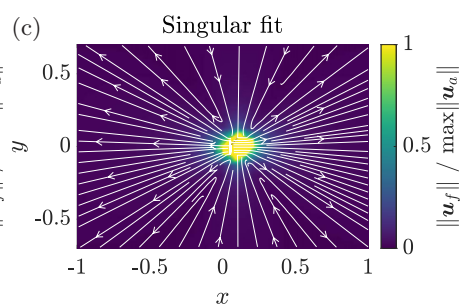

(f)

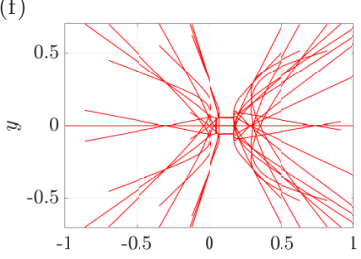

(i)

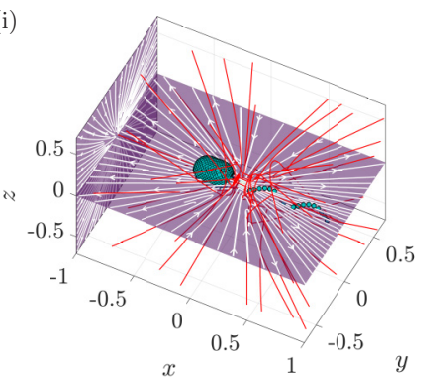

FIG. 1. Sample flow fields $(a, b, c)$ and streamlines (d, e, f) around a model bacterium positioned at the origin, with combined plots showing swimmer geometry and orientation $(\mathrm{g}, \mathrm{h}, \mathrm{i})$, where axis scales are in units of flagellum length. Panels (a, d, g) correspond to the original flow field $\boldsymbol{u}_{a}$, while panels (b, e, h) show $\boldsymbol{u}_{f}$ as generated using the fitted representation. Panels (c, f, i) show the results of fitting singular coincident Stokeslet and rotlet dipoles to the original flow field. The simple four-component regularized representation can be seen to qualitatively reproduce both the flow field and complex streamlines around the swimmer, with the rotational streamlines in particular unable to be well-approximated by the simple singular model. The magnitude of the fluid velocity relative to the maximum magnitude of $\boldsymbol{u}_{a}$ is shown in color in panels (a, b, c, g, h, i). Slices in panels $(\mathrm{a}, \mathrm{b}, \mathrm{c})$ are taken through $z=0$, with streamlines in panels $(\mathrm{d}, \mathrm{e}, \mathrm{f})$ projected onto this plane. Flow field slices in panels $(\mathrm{g}, \mathrm{h}, \mathrm{i})$ correspond to $z=0$ and $x=-1$.

over time $t$ as

$$
\frac{d \boldsymbol{X}^{k}}{d t}=\boldsymbol{U}^{\text {self }}+\boldsymbol{U}^{\text {others }}
$$

where $\boldsymbol{U}^{\text {others }}$ is the velocity field generated by the other swimmers, averaged over the locations of the regularized singularities in the representation of the $k$ th swimmer. Derived from the force and torque-free conditions appropriate for a neutrally buoyant microswimmer, analogous expressions hold for the angular velocity of the swimmers, as derived in previous works [35,38], given explicitly as

$$
\frac{d \boldsymbol{e}^{k}}{d t}=\mathbf{\Omega}^{\text {others }} \times \boldsymbol{e}^{k},
$$

where $\boldsymbol{e}^{k}$ is a body-fixed orientation vector and $\boldsymbol{\Omega}^{\text {others }}$ is the angular velocity calculated from the velocity field induced by the other swimmers, averaged over the locations of the regularized singularities. Where stated in the text, swimmer orientation is augmented with the effects of 


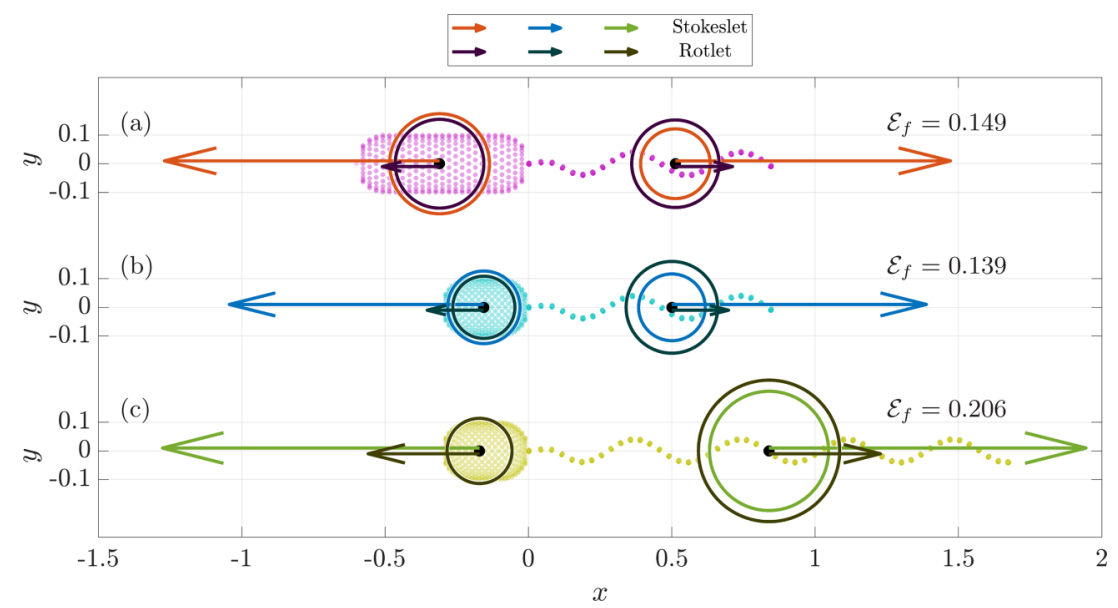

FIG. 2. Schematic of fitted swimmer representations and their associated geometries. Here, axis scales are in units of the flagellum length corresponding to case (b), with dots denoting points on the bacterium, while arrows correspond to strengths in both magnitude and direction, where the center and radius of the attached circles are the associated locations $\boldsymbol{x}_{0}^{k}$ and regularization parameters, with values given in Table I. We note that the regularized Stokeslet-rotlet pairs are positioned approximately at the geometric centers of the swimmer body and helix, with this observation apparently robust to considerable variations in morphological scales. Arrows are shown offset from their true positions for visual clarity, and strengths are uniformly rescaled for visualization. Relative measures of the size of the residual flow, $\mathcal{E}_{f}$ as defined in the main text, are also reported.

rotational noise, with simulations being repeated 1000 times to appropriately represent the inherent stochasticity.

When first considering pairwise dynamics, we initialize two swimmers, referred to as predator and prey, with their axes of symmetry lying in a common plane, without loss of generality the $x y$ plane of a Cartesian coordinate system centered on the head-tail junction of the prey swimmer, as in Figs. 1 and 2. Here, we position the predatory swimmer at $(x, y, 0)$ and align the prey swimmer along the $x$ axis as shown in Figs. 3(a) and 3(b). The $x y$-dependent initial orientation of the predatory swimmer is defined such that a collision of the swimmer's head-tail junctions would take place in the absence of any hydrodynamic interactions or rotational diffusion, and may be determined via elementary geometry as a function of relative swimmer speed and initial position. When later considering a broader range of initial conditions, we orient the swimmers as above then translate the predatory swimmer from $(x, y, 0)$ to $(x, y, c)$, equivalent to considering a range of initial out of plane orientations of the predator. For large values of $|c|$, we note that the probability of a collision from any given initial configuration $(x, y, c)$ approaches zero, with the predator effectively facing away from the prey. In practice, collisions are rarely observed when $|c|$ is greater than the body radii of the two swimmers.

For the purpose of detecting collisions when simulating swimmer motion, we represent each swimmer by a sphere of radius $\epsilon_{S}^{2}$ centered at $x_{0}^{2}$, which are, respectively, the regularization parameter and position of the regularized Stokeslet that is a posteriori coincident with the swimmer body in Fig. 2. A collision between two swimmers is then defined as a collision between their respective spheres, with the qualitative results presented in this work being insensitive to the details of this definition.

\section{RESULTS}

\section{A. Swimmer representation}

Informed by the character of the flow field and consistent with an approximate far-field force dipole representation, we approximate the computed flow field by a collection of two regularized 


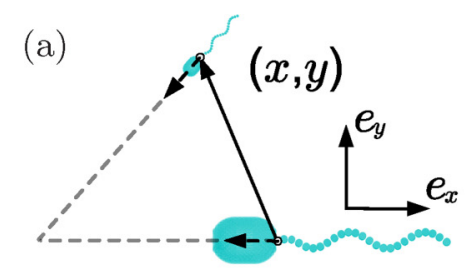

(c)

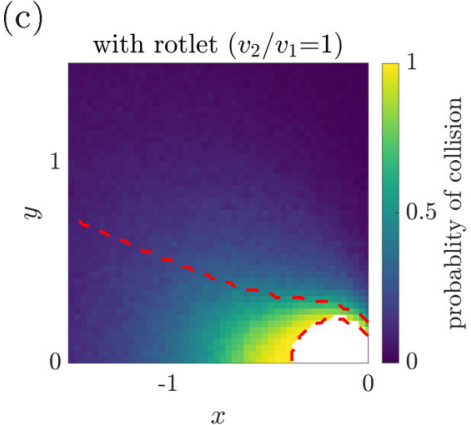

(e)

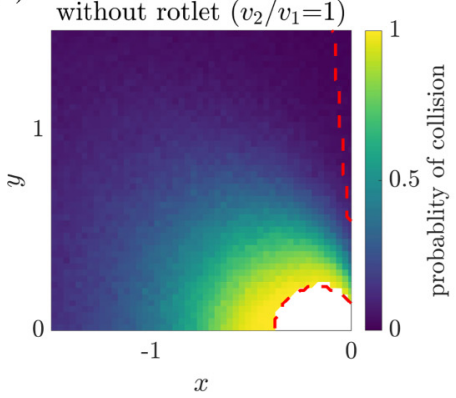

(b)

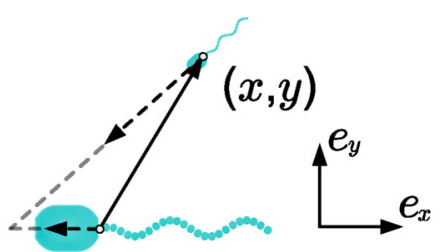

(d)

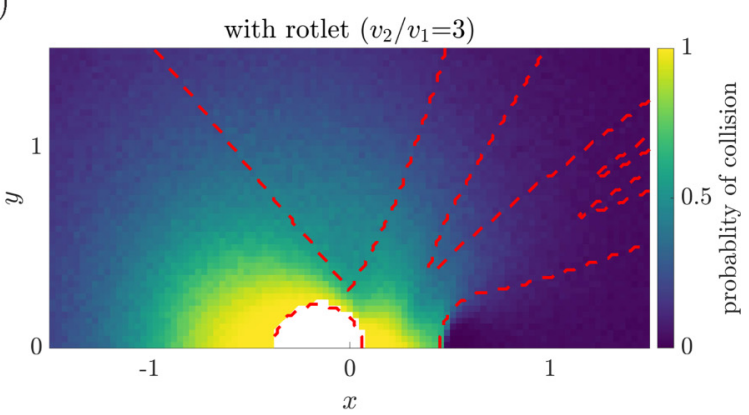

(f)

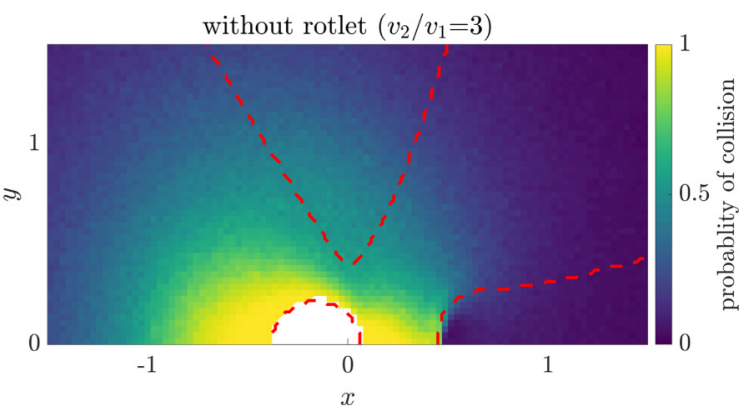

FIG. 3. Predator-prey collision dynamics, as computed with (c, d) and without (e, f) the effects of regularized rotlets, including and excluding rotational diffusion. $(a, b)$ Definitions of predator-prey configuration space for relatively slow (a) and fast (b) predators, with orientations defined such that collision would occur in the absence of hydrodynamic interactions or noise, and swimmer headings initially coplanar. For each initial configuration we simulate the unconstrained motion of the predator and prey until eventual collision or separation. In panels (c-f) we report the probability of a collision event occurring from a given initial configuration, with dashed red curves showing the boundaries of deterministic collision/separation regions in the absence of Brownian noise. Rotational diffusion can be seen to minimally impact on the qualitative dynamics, with both relative speed and swimmer representation dominating the predatory behaviors. In particular, we see that the inclusion of the regularized rotlet terms greatly reduces the prevalence of collisions in this case, while increased predator speed increases collision likelihood. Configurations in which swimmers overlap initially are shown white.

Stokeslets and two regularized rotlets, noting that the regularized rotlet decays more rapidly than the regularized Stokeslet as $\boldsymbol{r}$ approaches infinity. This representation, making use of regularized singularities as in the work of Ishimoto et al. [36], in particular, may enable the capturing of the rotational effects of the flagellum and counter-rotating body via the regularized rotlets, absent from previous simple descriptions of the flow field around a bacterium. Informed by the approximate symmetry of the bacterium about the axis of its flagellum, we constrain the positions of the regularized singularities to be along this midline, and, seeking further simplicity, impose that the representation consists only of coincident regularized Stokeslet-rotlet pairs with shared locations $\boldsymbol{x}_{0}^{k}$. Here and throughout, $k=1,2$ indexes the pairs of regularized singularities, and we note that 
TABLE I. Fitted representation parameters corresponding to the bacterial morphologies of Fig. 2. Owing to the approximate axial symmetry, we report only the $x$ coordinate of the regularized Stokeslet-rotlet pairs $\boldsymbol{x}_{0}^{k}$, denoted here as $x_{0}^{k}$ for $k=1,2$, and analogously for Stokeslet and rotlet strengths. Here, $k=1$ corresponds to the regularized singularities located in $x>0$ in Fig. 2.

\begin{tabular}{|c|c|c|c|c|c|c|c|c|}
\hline Case & $x_{0}^{1}$ & $x_{0}^{2}$ & $\epsilon_{S}^{1}$ & $\epsilon_{R}^{1}$ & $\epsilon_{S}^{2}$ & $\epsilon_{R}^{2}$ & $f^{1}$ & $m^{1}$ \\
\hline $\mathrm{a}$ & 0.51 & -0.31 & 0.12 & 0.15 & 0.17 & 0.15 & $3.2 \times 10^{-4}$ & $0.66 \times 10^{-4}$ \\
\hline $\mathrm{b}$ & 0.50 & -0.16 & 0.12 & 0.16 & 0.13 & 0.11 & $3.0 \times 10^{-4}$ & $0.66 \times 10^{-4}$ \\
\hline $\mathrm{c}$ & 0.84 & -0.17 & 0.21 & 0.25 & 0.11 & 0.13 & $3.7 \times 10^{-4}$ & $1.29 \times 10^{-4}$ \\
\hline
\end{tabular}

their corresponding strengths and regularization parameters may be chosen independently. Denoting the strengths of these regularized Stokeslets and rotlets by $\boldsymbol{f}^{k}$ and $\boldsymbol{m}^{k}$, respectively, imposing the force and torque-free conditions on this representation of the swimmer yields the simple constraints $\boldsymbol{f}^{(2)}=-\boldsymbol{f}^{(1)}$ and $\boldsymbol{m}^{(2)}=-\boldsymbol{m}^{(1)}$, with the torque balance being measured about an arbitrary inertial frame reference point [41]. Given these conditions, we compute the positions $\boldsymbol{x}_{0}^{k}$, regularization parameters $\epsilon_{S}^{k}, \epsilon_{R}^{k}$, and strengths $\boldsymbol{f}^{k}$ and $\boldsymbol{m}^{k}$ via nonlinear least-squares fitting of the flow field, utilizing the inbuilt MATLAB routine lsqnonlin. The computed optimal configuration for this bacterial morphology is illustrated in Fig. 2(b), with fitted parameters given in Table I. Consistent with the approximate symmetry of the time-averaged swimmer, we see the alignment of the regularized singularities along the centreline, as prescribed, with their strength vectors $\boldsymbol{f}^{k}$ and $\boldsymbol{m}^{k}$ oriented along the same axis. The proximity of the regularized Stokeslets gives rise to a far-field flow resembling that of a force dipole, and similarly the pair of regularized rotlets is reminiscent of a torque dipole.

Though we fit a regularized singularity representation to only the time-averaged flow around the virtual swimmer, throughout its motion the bacterium undergoes minimal yawing along its average trajectory. This is due to the approximate rotational symmetry of the swimmer about the axis of its helix, broken only by the chirality and thus slight asymmetry of the helical driver. Hence, an isolated bacterial swimmer may be well-represented as a time-independent collection of only four regularized singularities, moving with a constant linear velocity. This is in contrast to analogous approximations of the planar flagellar beating of human spermatozoa [36], which necessarily include the motion of the regularized singularities relative to the average position of the swimmer, and moreover the additional complexity of time-dependent strengths of the regularized point forces used. Our proposed representation therefore recovers the simplicity of the most basic force-dipole models of bacterial swimming, while improving upon the latter's limited capabilities to capture qualitative details of the complex flow field surrounding the bacterium.

\section{B. Near-field accuracy}

Qualitative comparisons of the original flow field with that produced by the proposed representation, each shown in Fig. 1, evidence a remarkable validity of this four-component model, with the characteristics of the flow appearing well-captured by our simple representation and in stark contrast to a fitted singular force and torque dipole representation. Similar consideration of the streamlines generated by the flows highlights analogous strong agreement between the complex character of the cyclical streamlines of the time-averaged flow and those generated by our approximate model. Introducing the energylike measure $\mathcal{E}(\boldsymbol{u}):=\int \boldsymbol{u}^{2} d V$ of a flow $\boldsymbol{u}$, where the integral is over the computational domain, which excludes points internal to the swimmer, and is computed in practice as a discretized sum over the $2.5 \times 10^{4}$ field points at which the flow is evaluated, we quantitatively evaluate the quality of the swimmer representation by considering the energy of the residual flow. Denoting the flow due to the fitted regularized representation by $\boldsymbol{u}_{f}$, we define the relative energy of the residual as $\mathcal{E}_{f}:=\mathcal{E}\left(\boldsymbol{u}_{a}-\boldsymbol{u}_{f}\right) / \mathcal{E}\left(\boldsymbol{u}_{a}\right)=0.139$, where $\boldsymbol{u}_{a}$ again denotes the time-averaged flow field computed via the boundary element method and the units are relative to the flagellum 
rotation frequency and the flagellum length of case b) in Fig. 2. In comparison, the approximation via singular Stokeslet and rotlet dipoles yields a relative residual energy of $\mathcal{E}_{s}=0.893$, with the proposed representation therefore being a marked improvement over previous singularity models as a means of near-field flow approximation. Further, considering a generalised representation of two regularized Stokeslets, of which a singular force dipole is a special case, yields a fitted configuration with associated residual energy of $\mathcal{E}_{f}=0.718$. Thus, despite being optimal amongst force dipole models, this reduced representation still yields significantly reduced near-field accuracy in comparison to the proposed four-component representation.

\section{Robustness to morphological variation}

Remarkably, for this sample bacterial morphology the regularized singularities are each approximately positioned at the midpoints of the body and the helix, suggesting that this approximation of the flow field is intuitively tied to the swimmer geometry. Sampling further from the diverse range of morphological scales exhibited by bacteria, though remaining restricted to those whose bodies are axisymmetric, we repeat the flow-field simulation and subsequent fitting process for model bacteria with either the body length or the flagellum length doubled relative to our original swimmer. With these morphologies and their accompanying fitted regularized singularities shown in Fig. 2, we see reproduced the approximate correspondence between the fitted positions $\boldsymbol{x}_{0}^{k}$ and the swimmer geometry, suggesting that this simple intuitive relation holds in more generality.

Further, calculations of flow-field accuracy in the case of these elongated morphologies again yields low residual energies, as reported in Fig. 2. Thus, the proposed representation of bacterial swimmers as a collection of four regularized singularities appears robust to realistic morphological heterogeneity in this model context, as does the approximate coincidence of the fitted locations of regularized singularities and the geometric centers of both the swimmer body and its helix.

\section{Hydrodynamics and predator-prey interaction}

We illustrate the significance of capturing near-field hydrodynamics by simulating the idealized predator-prey dynamics of two swimmers, incorporating hydrodynamic interactions via the proposed representation of bacterial swimming and focusing in particular on swimmer-swimmer collisions. As detailed in Sec. II C, we follow the approach of Ishimoto and Gaffney [35], extending their framework to include the contributions of regularized rotlets. In particular, the predator-prey simulations use a nondimensional model, obtained via a mass scale such that, without loss of generality, the nondimensional fluid viscosity is unity, the lengthscale is a typical flagellum length of the E. coli prey $(10 \mu \mathrm{m}[42])$, and the timescale is given by a typical E. coli flagellar rotation frequency $(100 \mathrm{~Hz}$ [43]), with the resulting average free-space swimming speed predicted by the simulation denoted by $v_{1}$. Then, as previously, the E. coli prey flagellum radius, in nondimensional units, is 0.003 , with a cell body of length 0.3 and radius 0.1 . The relative scales between predator and prey are then informed by those of the canonical predatory bacterium, B. bacteriovorus, which is modeled as having a reduced size compared to the prey, with dimensions scaled down by a factor of three and noting that the proposed swimmer representation accounts for finite-size effects, in contrast to basic dipole swimmers. Furthermore, and motivated by the wide range of swimming speeds and flagellar rotation rates exhibited by bacterial predators and bacteria more generally $[32,44-46]$, denoting such predatory speeds by $v_{2}$, we consider predator-prey pairs with both $v_{2} / v_{1}=1$ and $v_{2} / v_{1}=3$, corresponding to slow and fast predators, respectively, with flagellar rotation rates of 300 and $900 \mathrm{~Hz}$.

We first consider swimmers with both their headings and relative positions initially in a plane spanned by unit vectors $\boldsymbol{e}_{x}$ and $\boldsymbol{e}_{y}$, as shown in Figs. 3(a) and 3(b), with position-dependent initial orientations taken such that the swimmers would collide in the absence of any hydrodynamic interactions or Brownian noise, as detailed in Sec. IIC. Sampling from a range of initial conditions, we simulate the unconstrained three-dimensional motion of the two swimmers from a multitude of 
relative initial positions until their eventual collision or significant separation, with each swimmer represented by the four-component configuration of regularized singularities fit to their morphology and flagellar rotation rate.

The boundaries between regions of the configuration space resulting in deterministic collision and in deflection are plotted on Figs. 3(c) and 3(d) as red dashed curves, with the connected components in each plot that contain the bottom left-most point of the configuration space corresponding to eventual collision. Clearly visible in the slow-predator case [Fig. 3(c)] is the vast proportion of initial conditions from which collision events are unobserved, in contrast to the dynamics exhibited by the fast-predator [Fig. 3(d)]. In the latter case, the increased swimming speed of the faster predator appears to promote collisions across a broad range of initial configurations, while in the equal-speed case we hypothesize that the predator is simply scattered by the high-magnitude rotational flows generated by the counterrotation of the prey body and helix.

We examine this hypothesis more closely by repeating the simulations of predator-prey motion utilizing newly fitted swimmer representations containing only regularized Stokeslets, with no rotlets, which are thus an optimum amongst force dipole models. The analogous pairwise behaviors resulting from these simulations are shown in Figs. 3(e) and 3(f), from which we note qualitative differences between resultant behaviors when including or neglecting regularized rotlet terms. In particular, from the initial conditions considered in Fig. 3, the noted pairwise attraction of force-dipole swimmers [1] appears dominated by the rotational flow contributions of the regularized rotlets, more generally suggesting that neglecting detailed hydrodynamic interactions in favor of more basic representations of bacterial motion has the potential to limit the complexity and accuracy of predicted dynamics, with such interactions emergent from the presented simple swimmer representation.

This conclusion is further evidenced by considering a broader range of initial conditions, as detailed in Sec. IIC. Translating the predatory cell to $z=c$, shown in Fig. 4 for $z= \pm 0.1$, we observe that the dynamics in the absence of rotlet terms, shown in Figs. 4(c) and 4(d), are independent of the sign of the initial $z$ coordinate, with the inherent chirality of the swimmers not being captured by such models. Indeed, Figs. 4(a) and 4(b) highlight that the rotlet terms of the proposed representation yield significant differences between the cases of $z=c$ and $z=-c$, as may be expected due to the signed rotation of the helical drivers of the idealized bacteria. Hence, overall, we have seen that the most basic models of swimmer hydrodynamics can significantly overestimate [Fig. 3(c) versus 3(e)] or significantly underestimate [Fig. 4(a) versus 4(c)] the prevalence of bacterial collision, and thus contact-mediated predation, in the absence of rotational diffusion depending on the initial configuration of the swimmers.

\section{E. Effects of rotational diffusion on collision dynamics}

Finally, we examine the effects of rotational diffusion on the collision behaviors, reasoned to be significant by Drescher et al. [26] from force-dipole representations of bacterial swimmers. Rotational diffusion coefficients of the swimmers are taken as in Drescher et al. [26] for E. coli for both predator and prey bacteria, with this choice of coefficient qualitatively reproducing the behaviors of B. bacteriovorus despite its reduced size (Supplemental Material movies in Ref. [32]). Repeating each simulation 1000 times from the same initial conditions as the above deterministic simulations, in Figs. 3 and 4 we show the empirically estimated probability of collision from a given configuration as heatmaps, with the standard error of these estimates being bounded by $1.6 \%$ and scaling with the reciprocal of the square root of the number of repeats. Perhaps surprisingly, we see that the deterministic simulations have generally well-captured the broad features of the diffusive simulations, with regions of configuration space resulting in collisions in the former being typically associated with regions of higher probability of collision in the latter. Further, except in the far field, we note that the effects of including the regularized rotlet terms remain significant even with the inclusion of rotational noise, though this relation expectedly becomes weaker with increasing distance from the prey bacterium. Therefore, this evidences that, even at larger cell separations, 
(a)

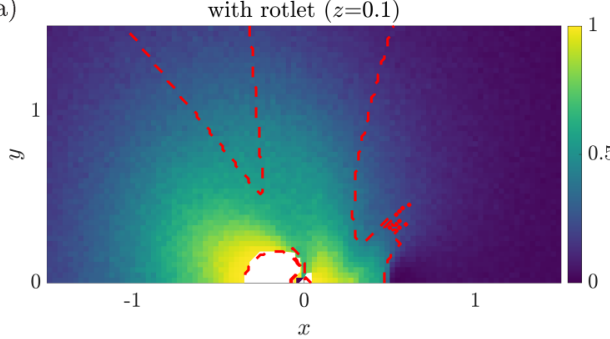

(c)

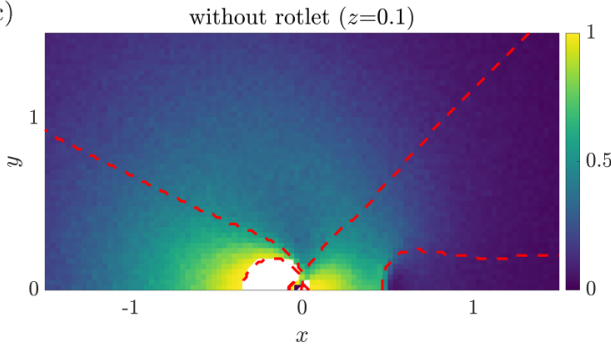

(b)

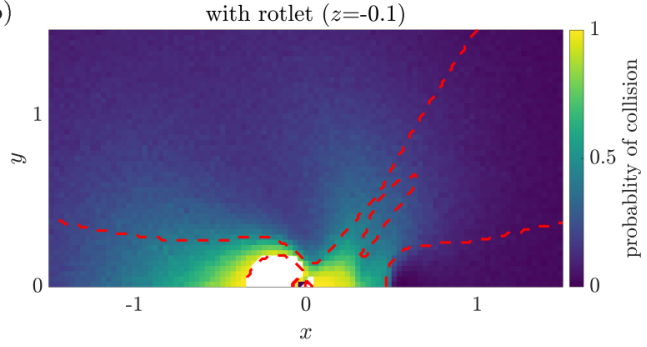

(d)

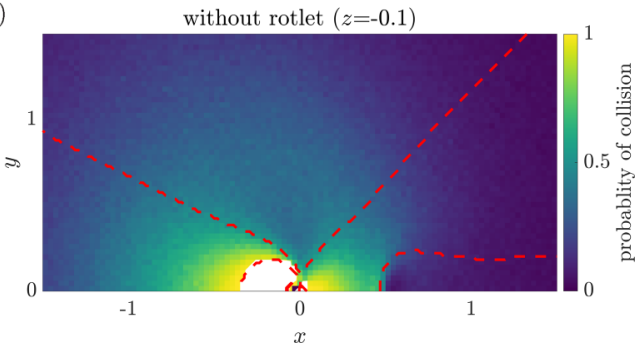

FIG. 4. Predator-prey collision dynamics, as computed with (a,b) and without (c, d) the effects of regularized rotlets, including and excluding rotational diffusion and initially with $z=0.1$ and $z=-0.1$ for panels $(a, c)$ and $(b, d)$, respectively. For each initial configuration we simulate the unconstrained motion of the predator and prey until eventual collision or separation, with $v_{2} / v_{1}=3$. The probability of a collision event occurring from a given initial configuration is represented by color, with dashed red curves showing the boundaries of deterministic collision/separation regions in the absence of Brownian noise. As in the case of $z=0$ in Fig. 3, rotational diffusion can be seen to minimally impact on the qualitative dynamics, with swimmer representation dominating the predatory behaviors. Most notably, we see a qualitative difference between panels (a) and (b), with only the rotlet terms successfully incorporating swimmer chirality. Unable to capture this chirality, we see that the Stokeslet-only models of (c) and (d) predict the same dynamics regardless of the sign of $z$. Configurations in which swimmers overlap initially are shown white.

hydrodynamics has a major impact on bacterial swimmer-swimmer interactions, in distinct contrast to general inferences from force-dipole models [26].

Finally, we briefly consider a potential summary statistic of interest, the overall probability of collision. Given prior knowledge of swimmer configuration, or a distribution of configurations, one may compute a measure of the overall collision probability by summing over the possible configurations, weighting by the prior. For example, taking the prior distribution to be uniform over the initial conditions considered here, averaging between $z=-0.15$ and $z=0.15$, the summary statistics for fast-predator models with and without rotlet terms are similar, at around $23.3 \%$ and $24.9 \%$, respectively. These summary statistics are therefore not representative of the significant differences observed in Figs. 3 and 4, highlighting that simple summary statistics are not a suitable proxy for the complex dynamics exhibited here.

\section{SUMMARY AND CONCLUSIONS}

In this work we have presented a refined representation of the flow field around a flagellated bacterial swimmer in the laboratory frame, utilizing regularized point forces and torques to simply capture the complex qualitative features of fluid flow. By direct comparison, we have evidenced the increased near-field accuracy afforded by this model over optimal singular and regularized dipole models, at the cost of additional terms in the swimmer representation. Nonetheless, retained in our representation is a simplicity that is reminiscent of these far-field dipole models, enabling the straightforward incorporation of highly resolved near-field hydrodynamics into large-scale simulations of active matter at little computational cost, for example, in the study of bacterial 
turbulence or the interactions of magnetically driven microswimmers [8,12]. The inclusion of regularized rotlet terms in the swimmer representation was seen to dramatically increase near-field accuracy, well-capturing both the qualitative and quantitative features of the complex spiraling flows around a model bacterium.

Further, we have noted that the omission of rotlet terms in pairwise dynamics can have a profound effect on overall behavior and any resulting predictions. Motivated by bacterial predator-prey interactions, by the direct simulation of two morphologically and dynamically distinct swimmers in close proximity we have evidenced that the characteristic hydrodynamics captured by our time-invariant four-component representation give rise to a rich pairwise dynamics, qualitatively distinct from those obtained with force-dipole models that have nonetheless seen widespread use. Irrespective of the presence of rotational diffusion, the behaviors of collision and escape between model predator and prey swimmers are greatly impacted by the explicit inclusion of the rotational motion of the swimmer and its propulsive helix. This signifies the relative importance of considering hydrodynamic interactions in models of bacterial swimming, with particular reference to swimmer chirality, which may not be reliably replaced by rotational noise alone. Additionally, these explorations suggest a potential configuration-dependent overestimation or underestimation of bacterial predation by simple force-dipole models that may be of pertinence to more sophisticated computational and theoretical evaluations of predatory bacteria that include relevant physiological factors, such as the possible chemotaxis of predatory species, steric interactions between predator and prey, or the presence of multiple predator or prey bacteria. With multiple recent works having suggested and evaluated predatory bacteria for use in industry [47-49], we envisage that the refined hydrodynamic model presented in this work will enable higher fidelity theoretical investigation into the cell-level dynamics of these applications, in addition to forming the basis of models exploring the effects of environment heterogeneity and the impacts of viscosity on predation [32,50-52].

In agreement with intuition, though without rigorous justification a priori, we have noted the approximate geometric coincidence of optimally placed regularized singularities and the midpoints of swimmer bodies and flagella. This approximate relation arises from flow-field optimization rather than symmetry arguments, which would not allow for the observed imprecision. Nevertheless, this relation does appear to hold across a range of relative morphological scales, noting the restriction that we have considered only idealized polar swimmers in this study. The extension of such a relation to more complex bacterial morphologies represents a potential direction for future study, with the presented approach for swimmer representation readily extending to those containing additional regularized terms, which may be necessary in accurately modeling swimmers with elongated or irregular morphologies.

In summary, this study has emphasized the importance of the inclusion of detailed near-field hydrodynamics when seeking to accurately capture the complex behaviors of bacterial active matter, with pairwise interactions evidenced to be significantly affected by their omission. Further, this work has constructed a simple four-component model of a bacterial swimmer, facilitating the inclusion of near-field flows into existing approaches and frameworks at little computational expense via an intuitive yet accurate regularized representation.

The research materials supporting this article have been made available at [53].

\section{ACKNOWLEDGMENTS}

B.J.W. is supported by the UK Engineering and Physical Sciences Research Council (EPSRC), Grant No. EP/N509711/1. K.I. is supported by JSPS-KAKENHI for Young Researchers (Grant No. 18K13456), JST, and PRESTO Grant No. JPMJPR1921. Elements of the numerical simulations were performed within the cluster computer system at the Research Institute for Mathematical Sciences (RIMS) and Institute for Information Management and Communication (IIMC), Kyoto University. 
[1] E. Lauga and T. R. Powers, The hydrodynamics of swimming microorganisms, Rep. Prog. Phys. 72, 096601 (2009).

[2] D. J. Smith, E. A. Gaffney, J. R. Blake, and J. C. Kirkman-Brown, Human sperm accumulation near surfaces: A simulation study, J. Fluid Mech. 621, 289 (2009).

[3] J. Elgeti, U. B. Kaupp, and G. Gompper, Hydrodynamics of sperm cells near surfaces, Biophys. J. 99, 1018 (2010).

[4] B. J. Walker, R. J. Wheeler, K. Ishimoto, and E. A. Gaffney, Boundary behaviours of Leishmania mexicana: A hydrodynamic simulation study, J. Theor. Biol. 462, 311 (2019).

[5] M. Leoni and T. B. Liverpool, Swimmers in Thin Films: From Swarming to Hydrodynamic Instabilities, Phys. Rev. Lett. 105, 238102 (2010).

[6] P. T. Underhill, J. P. Hernandez-Ortiz, and M. D. Graham, Diffusion and Spatial Correlations in Suspensions of Swimming Particles, Phys. Rev. Lett. 100, 248101 (2008).

[7] M. C. Marchetti, J. F. Joanny, S. Ramaswamy, T. B. Liverpool, J. Prost, M. Rao, and R. A. Simha, Hydrodynamics of soft active matter, Rev. Mod. Phys. 85, 1143 (2013).

[8] J. Dunkel, S. Heidenreich, K. Drescher, H. H. Wensink, M. Bär, and R. E. Goldstein, Fluid Dynamics of Bacterial Turbulence, Phys. Rev. Lett. 110, 228102 (2013).

[9] D. Lopez and E. Lauga, Dynamics of swimming bacteria at complex interfaces, Phys. Fluids 26, 071902 (2014).

[10] D. O. Pushkin and J. M. Yeomans, Stirring by swimmers in confined microenvironments, J. Stat. Mech.: Theory Exp. (2014) P04030.

[11] D. O. Pushkin and J. M. Yeomans, Fluid Mixing by Curved Trajectories of Microswimmers, Phys. Rev. Lett. 111, 188101 (2013).

[12] F. Meng, D. Matsunaga, and R. Golestanian, Clustering of Magnetic Swimmers in a Poiseuille Flow, Phys. Rev. Lett. 120, 188101 (2018).

[13] A. Baskaran and M. C. Marchetti, Statistical mechanics and hydrodynamics of bacterial suspensions, Proc. Natl. Acad. Sci. U.S.A. 106, 15567 (2009).

[14] A. Jepson, V. A. Martinez, J. Schwarz-Linek, A. Morozov, and W. C. K. Poon, Enhanced diffusion of nonswimmers in a three-dimensional bath of motile bacteria, Phys. Rev. E 88, 041002 (2013).

[15] G. L. Miño, J. Dunstan, A. Rousselet, E. Clément, and R. Soto, Induced diffusion of tracers in a bacterial suspension: Theory and experiments, J. Fluid Mech. 729, 423 (2013).

[16] E. Lushi, H. Wioland, and R. E. Goldstein, Fluid flows created by swimming bacteria drive selforganization in confined suspensions, Proc. Natl. Acad. Sci. U.S.A. 111, 9733 (2014).

[17] H. Wioland, E. Lushi, and R. E. Goldstein, Directed collective motion of bacteria under channel confinement, New J. Phys. 18, 075002 (2016).

[18] B. Zhang, Y. Ding, and X. Xu, Active suspensions of bacteria and passive objects: A model for the near field pair dynamics, arXiv:2002.04693.

[19] R. Cortez, L. Fauci, and A. Medovikov, The method of regularized Stokeslets in three dimensions: Analysis, validation, and application to helical swimming, Phys. Fluids 17, 031504 (2005).

[20] B. Rodenborn, C.-H. Chen, H. L. Swinney, B. Liu, and H. P. Zhang, Propulsion of microorganisms by a helical flagellum, Proc. Natl. Acad. Sci. U.S.A. 110, E338 (2013).

[21] B. Liu, K. S. Breuer, and T. R. Powers, Helical swimming in Stokes flow using a novel boundary-element method, Phys. Fluids 25, 061902 (2013).

[22] H. Flores, E. Lobaton, S. Mendezdiez, S. Tlupova, and R. Cortez, A study of bacterial flagellar bundling, Bull. Math. Biol. 67, 137 (2005).

[23] S. E. Spagnolie and E. Lauga, Hydrodynamics of self-propulsion near a boundary: Predictions and accuracy of far-field approximations, J. Fluid Mech. 700, 105 (2012).

[24] E. Tjhung, M. E. Cates, and D. Marenduzzo, Contractile and chiral activities codetermine the helicity of swimming droplet trajectories, Proc. Natl. Acad. Sci. U.S.A. 114, 4631 (2017).

[25] R. Cortez, The method of regularized Stokeslets, SIAM J. Sci. Comput. 23, 1204 (2001).

[26] K. Drescher, J. Dunkel, L. H. Cisneros, S. Ganguly, and R. E. Goldstein, Fluid dynamics and noise in bacterial cell-cell and cell-surface scattering, Proc. Natl. Acad. Sci. U.S.A. 108, 10940 (2011). 
[27] H. Shum and J. M. Yeomans, Entrainment and scattering in microswimmer-colloid interactions, Phys. Rev. Fluids 2, 113101 (2017).

[28] J. Dölger, L. T. Nielsen, T. Kiørboe, and A. Andersen, Swimming and feeding of mixotrophic biflagellates, Sci. Rep. 7, 39892 (2017).

[29] A. J. T. M. Mathijssen, R. Jeanneret, and M. Polin, Universal entrainment mechanism controls contact times with motile cells, Phys. Rev. Fluids 3, 033103 (2018).

[30] P. Mueller and J.-L. Thiffeault, Fluid transport and mixing by an unsteady microswimmer, Phys. Rev. Fluids 2, 013103 (2017).

[31] S. Beck, D. Schwudke, E. Strauch, B. Appel, and M. Linscheid, Bdellovibrio bacteriovorus strains produce a novel major outer membrane protein during predacious growth in the periplasm of prey bacteria, J. Bacteriol. 186, 2766 (2004).

[32] H. Jashnsaz, M. Al Juboori, C. Weistuch, N. Miller, T. Nguyen, V. Meyerhoff, B. McCoy, S. Perkins, R. Wallgren, B. D. Ray, K. Tsekouras, G. G. Anderson, and S. Pressé, Hydrodynamic hunters, Biophys. J. 112, 1282 (2017).

[33] J. Pérez, A. Moraleda-Muñoz, F. J. Marcos-Torres, and J. Muñoz-Dorado, Bacterial predation: 75 years and counting! Environ. Microbiol. 18, 766 (2016).

[34] D. Negus, C. Moore, M. Baker, D. Raghunathan, J. Tyson, and R. E. Sockett, Predator Versus Pathogen: How does predatory Bdellovibrio bacteriovorus interface with the challenges of killing gram-negative pathogens in a host setting? Annu. Rev. Microbiol. 71, 441 (2017).

[35] K. Ishimoto and E. A. Gaffney, Hydrodynamic clustering of human sperm in viscoelastic fluids, Sci. Rep. 8, 15600 (2018).

[36] K. Ishimoto, H. Gadêlha, E. A. Gaffney, D. J. Smith, and J. Kirkman-Brown, Coarse-Graining the Fluid Flow Around a Human Sperm, Phys. Rev. Lett. 118, 124501 (2017).

[37] C. Pozrikidis, A Practical Guide to Boundary Element Methods with the Software Library BEMLIB (CRC Press, Boca Raton, FL, 2002).

[38] B. J. Walker, K. Ishimoto, and E. A. Gaffney, Pairwise hydrodynamic interactions of synchronized spermatozoa, Phys. Rev. Fluids 4, 093101 (2019).

[39] N. Dasgupta, S. K. Arora, and R. Ramphal, The flagellar system of Pseudomonas aeruginosa, in Pseudomonas, edited by J.-L. Ramos (Springer, Boston, MA, 2004), pp. 675-698.

[40] K. Ishimoto, Bacterial spinning top, J. Fluid Mech. 880, 620 (2019).

[41] C. Pozrikidis, Boundary Integral and Singularity Methods for Linearized Viscous Flow (Cambridge University Press, Cambridge, UK, 1992).

[42] L. Turner, W. S. Ryu, and H. C. Berg, Real-time imaging of fluorescent flagellar filaments, J. Bacteriol. 182, 2793 (2000).

[43] N. C. Darnton, L. Turner, S. Rojevsky, and H. C. Berg, On torque and tumbling in swimming Escherichia coli, J. Bacteriol. 189, 1756 (2007).

[44] C. Lambert, K. J. Evans, R. Till, L. Hobley, M. Capeness, S. Rendulic, S. C. Schuster, S.-I. Aizawa, and R. E. Sockett, Characterizing the flagellar filament and the role of motility in bacterial prey-penetration by Bdellovibrio bacteriovorus, Mol. Microbiol. 60, 274 (2006).

[45] Y. Magariyama, S. Sugiyama, K. Muramoto, Y. Maekawa, I. Kawagishi, Y. Imae, and S. Kudo, Very fast flagellar rotation, Nature 371, 752 (1994).

[46] S. Zhu, S. Kojima, and M. Homma, Structure, gene regulation and environmental response of flagella in Vibrio, Front. Microbiol. 4, 1 (2013).

[47] O. Olanya, B. Niemira, J. Cassidy, G. Boyd, and J. Uknalis, Pathogen reduction by predatory bacteria and survival of Bdellovibrio bacteriovorus and Escherichia coli on produce and buffer treated with low-dose gamma radiation, LWT 130, 109630 (2020).

[48] C. Herencias, S. Salgado-Briegas, and M. A. Prieto, Emerging horizons for industrial applications of predatory bacteria, in The Ecology of Predation at the Microscale (Springer International Publishing, Cham, 2020), pp. 173-194.

[49] M. J. Boileau, K. D. Clinkenbeard, and J. J. Iandolo, Assessment of Bdellovibrio bacteriovorus 109J killing of Moraxella bovis in an in vitro model of infectious bovine keratoconjunctivitis, Can. J. Vet. Res. 75, 285 (2011). 
[50] H. Im, H. Kwon, G. Cho, J. Kwon, S. Y. Choi, and R. J. Mitchell, Viscosity has dichotomous effects on Bdellovibrio bacteriovorus HD100 predation, Environ. Microbiol. 21, 4675 (2019).

[51] M. Petrenko, S. P. Friedman, R. Fluss, Z. Pasternak, A. Huppert, and E. Jurkevitch, Spatial heterogeneity stabilizes predator-prey interactions at the microscale while patch connectivity controls their outcome, Environ. Microbiol. 22, 694 (2020).

[52] H. Im, L. E. Bäcker, and R. J. Mitchell, Environmental and biotic factors impacting the activities of Bdellovibrio bacteriovorus, in The Ecology of Predation at the Microscale (Springer International Publishing, Cham, 2020), pp. 155-172.

[53] K. Ishimoto, E. A. Gaffney, and B. J. Walker, Data relating to Regularized representation of bacterial hydrodynamics, https://doi.org/10.5287/bodleian:Eo4J0dzDP (2020). 